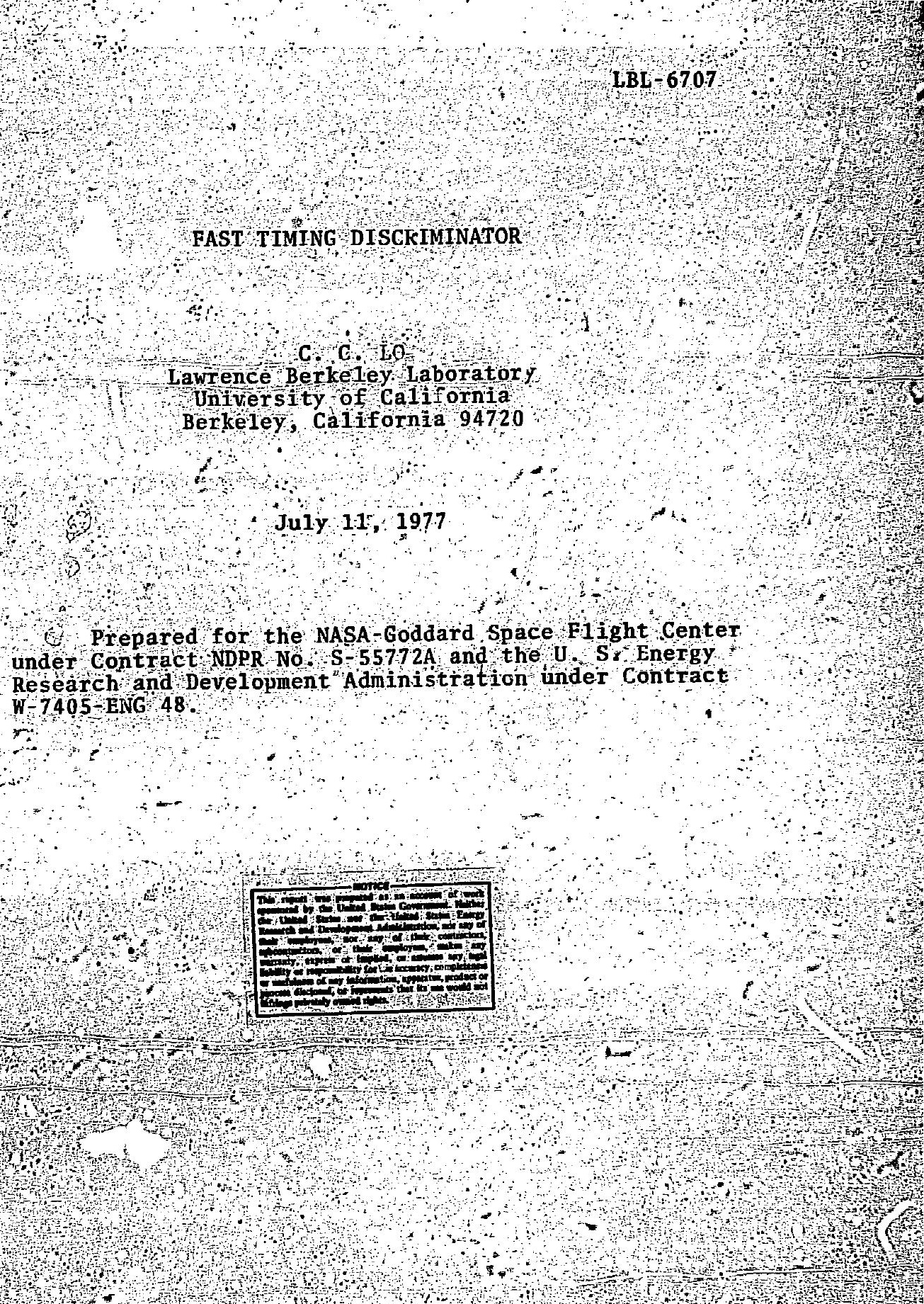

FAST TIMING DISGKIMINATOR 


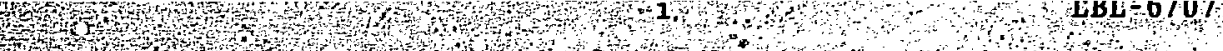

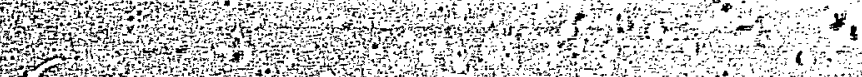

1. Introduction

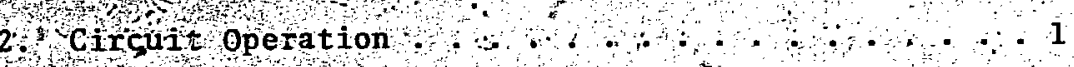

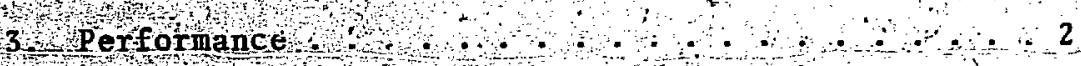

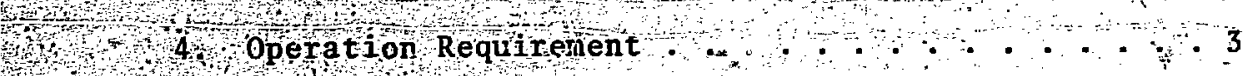

1. 5 , Acknow1edgments,

6 , Append $1 x+1$,

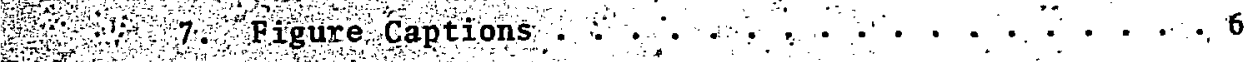

8.Appendix I \&

9. Patts list o. . . . . . . . . .18

W

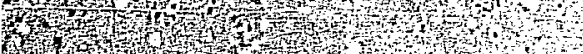

Whothes

Thx

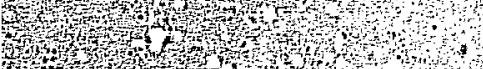

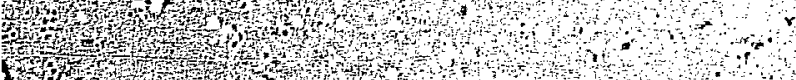


FAST TIMING DISCRIMINATOR

\author{
C. Clo \\ Lawrénce Berkeley Laboratory \\ University of California \\ Berkeley, California 94720
}

$$
\text { July } 11,1977
$$

\title{
Introduction
}

The processing of pl ases with very fast risetimes for timing purposes involves many problems because of the large equivalent bandwidths involved. For pulses with rîsetimes in the 150 ps range (and full widths at half maximum (FWHM) of $400 \mathrm{ps}$ ) bandwidths in excess of $1 \mathrm{GHz}$ are required. Furthermore, these very narrow pulses with current amplitúdes as smal1 as $1 \mathrm{~mA}$ carry very small charges ( $<10^{-12}$ coulomb), therefore, requiring very sensitive trigger circuits. The difficuity increases when timing characteristics in the picosecond range are sought expecialiy when a wide input signal ampiltude range causes a time-walk problem. The fast timing discriminator described here has atime-walk of approximately +75 ps over the input ishal range from 80 m to $3 \mathrm{~V}$

\section{Circint operiation}

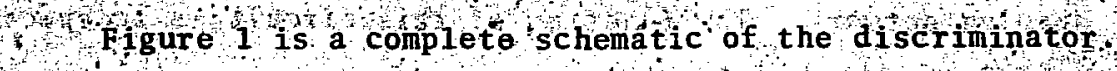
Signals enter at $\mathrm{PG}_{2}$ and $\mathrm{R}_{1}, \mathrm{R}_{2}, \mathrm{CR}_{1}-\mathrm{CR}_{2}$ serve as an amplitude initer for high amplitude puises The signal, is injected into 
the tunnel dode GR through transformed 1 , Bias on GR 1s set by $\mathrm{R}_{3}$ and $\mathrm{R}_{4}, \mathrm{R}$, being a ten turn potentioneter mounted on the front pand tor tow threshold adjustment Whit $\mathrm{R}_{4}, \mathrm{~s}$ ounted on the rear panel and used for adjust-

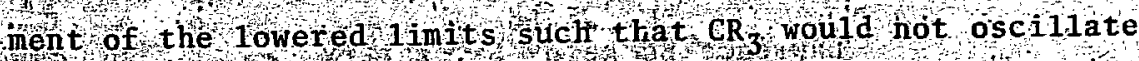

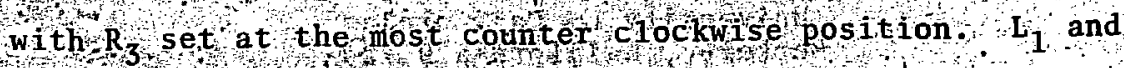

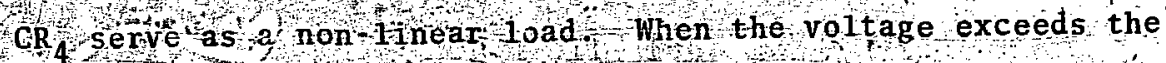

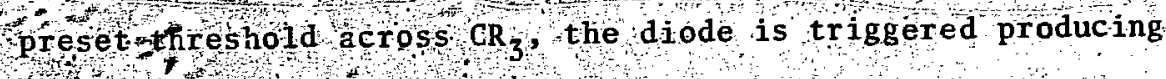
a pulse whosewidth is determined by $\mathrm{L}_{1}$ This pulse passes through $\mathrm{R}_{6}$, nd CR, to the, tunnel dode CR in the driver stage: When this dode ts triggered it produces a pulse which is used to drive the comparafor, $\mathrm{M}$ which, in turn, drives the five oùtput stages:

\section{Berformance}

If $w x+2$

since the signal pulse energy is below 1 x. $10^{-12}$ coulomb, $1 \mathrm{t}, \mathrm{s}$ ogicalto use a tunmel dode as trigger element Both tunne d iodestages are one-shot 1eading-edge-trigger-multiWhrators the walk charateristics of leading edge discriminators depend on the threshold setting of the discriminator

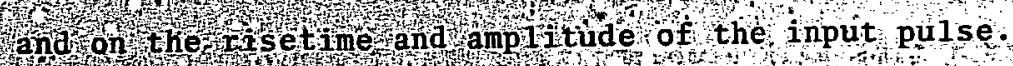

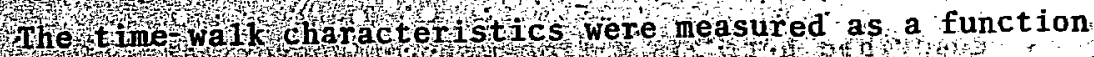

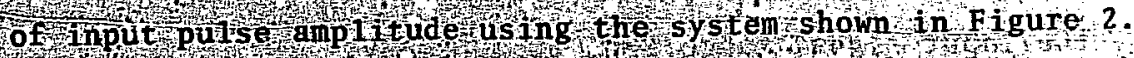

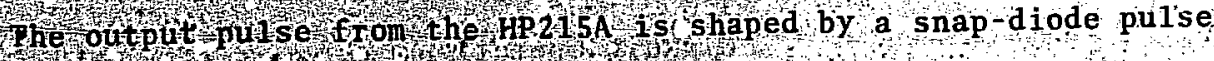

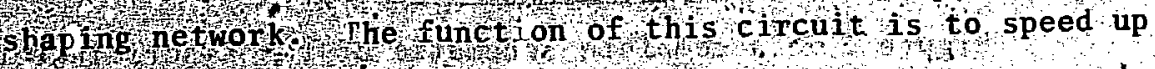

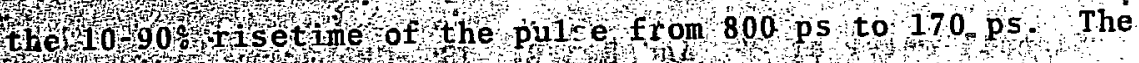

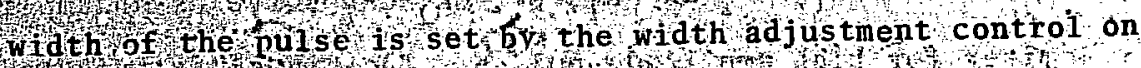
the HP pul se generatorexcept incase of the 250 ps púlse width 
where a clipping stub is used at the output of the snap diode shaperto dofferentiate the main pulse and provide a shorter pulse. E Fure $3 A$ and $4 A$ show the 400 ps and 250 ps pulse respectively. Figure $3 \mathrm{~B}$ and $4 \mathrm{~B}$ show the output pulse from discriuinator $B$ and $A$ respectively.:

Eor an input pulse having a risetime of 170 ps and a pulse width of too ps at the KWHM points, the time-walkover a dynamic range from $80 \mathrm{mV}$ to $3 \mathrm{~V}$ is $t 5 \mathrm{ps}$ For an input pulse having a risetime of 170 ps and pulse width of 250 ps at the FWH points, the time-walk over the same dynamic range is 150 ps : The time-walk characteristics as a functioi of amplitude are shown in Figs. 5 and 6 .

\section{Operation Requirement}

The discriminators are built in a singles width NIM module and require. $\pm 6 \mathrm{~V}$ and $+12 \mathrm{~V}$ to operate. The front paneI threshold adjustment sets the first tunnel diode stage bias; this adjustwill affect the time-walk in the low signal amplitude range. To obtain the best walk characteristic over the widest dynamic trange, the threshold setting should be set at the most stnsitive position ojust above the oscillation threshold of the tunne diode) The driver stage threshold bias does not normally need adjustment. For stable operation the discriminators should be powered at a 11times, Abrief sumary of likely fault conditions is given in Appendix $\mathrm{I}$

A print 1 ist of the discriminator is given in Appendix II 

Appendix 1:

Symptoms

Random output.

pulses without

input

No outpat pulse
Trouble Shooting
1. Front panel threshold setting too sensitive. (Readjust in a clockwise direction) 2. Driver stage bias too high.

1. Check power supply to the discriminators.

2. Check input pulse.

3. Use high-impedance wideband probe to trace the signal from, input to output, stage by stage:

4. Make proper adjustments or replace defective componients.

Excessive walk. 1. Check input pulse-width, amplitude and risetime making sure they meet the input specifications; $150-170 \mathrm{ps}, 10-90 \%$ pulse risetime with a pulse width of 400 ps at the FWHM points.

2. Tmproper threshold bias adjustment. 3. Improper driver bias adjustment. 


\section{Figure Captions}

1. Schematic diagram of the fast timing discriminator. System block diagram of the time-walk measuring system. 3A. 400 ps input signal pulse.

3B output pulse from discriminator $B$.

4A. 250 ps input signal pulse:

4B. Output pulse from discriminator $A$.

5. Discriminator time-walk characteristics as a function of the amplitude of a signal puise having a width of 400 ps at FWHM.

6. Discriminator time-waik characteristics as a function 4,4 of the anplitude of a signal pulse having a width of 250 ps at FWHM

7. Front panel of the fast timing discriminator.

8. Rear paned of the fast timing discriminator.

9. Wring side of the printed circuit board of the fast timing discriminator.

10. Component side of the printed circuit board of the fast timing discriminator. 


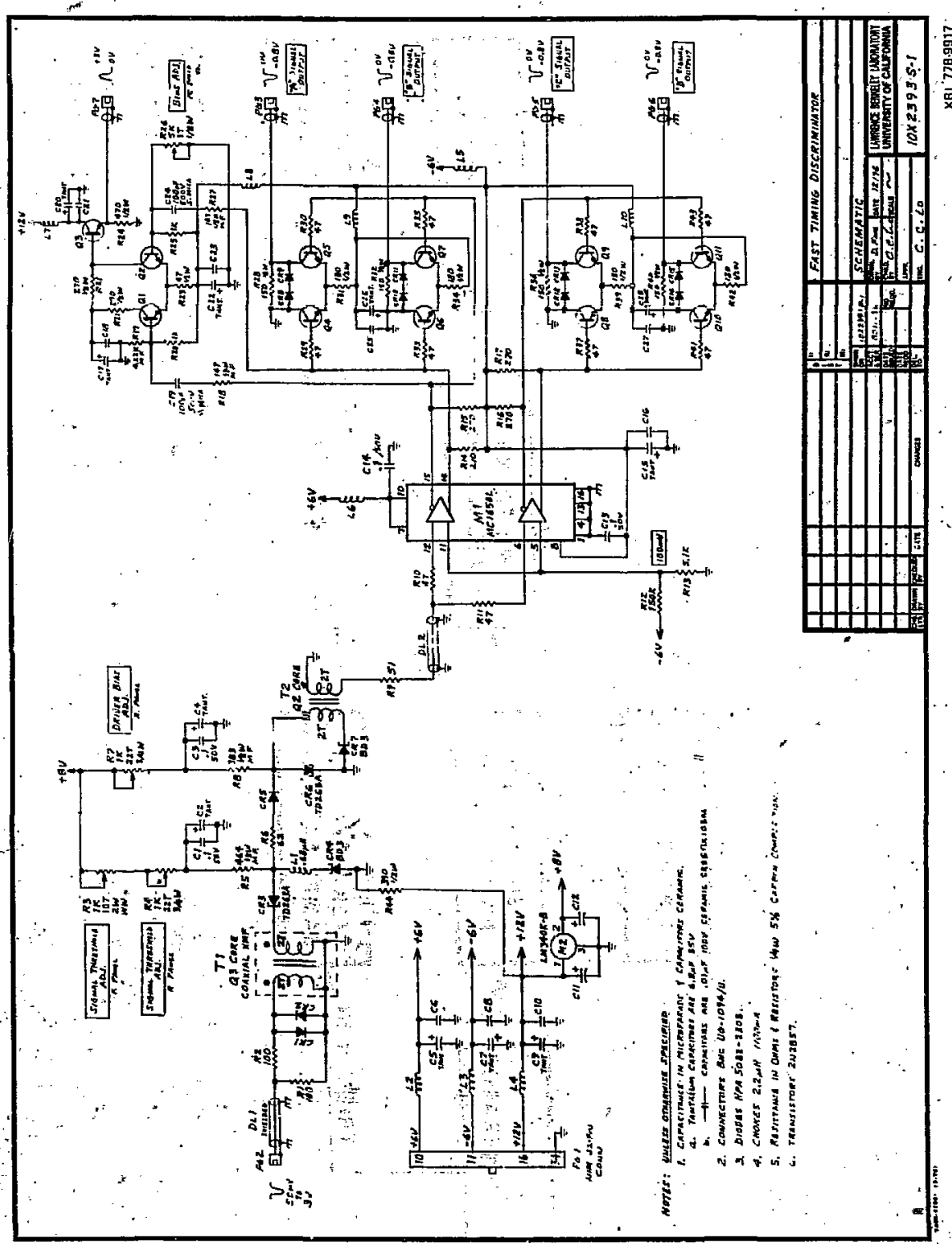

Fig. 1 


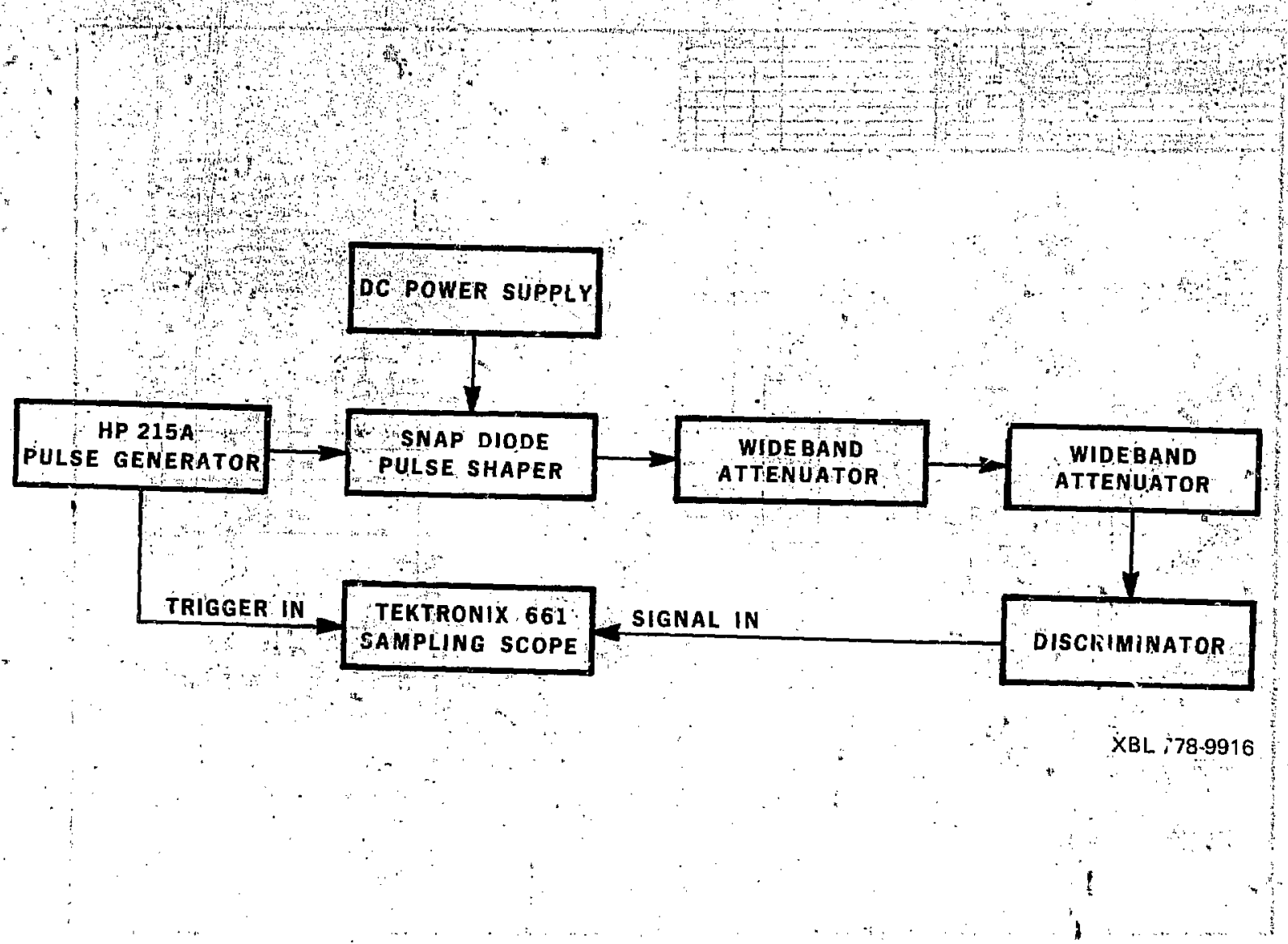




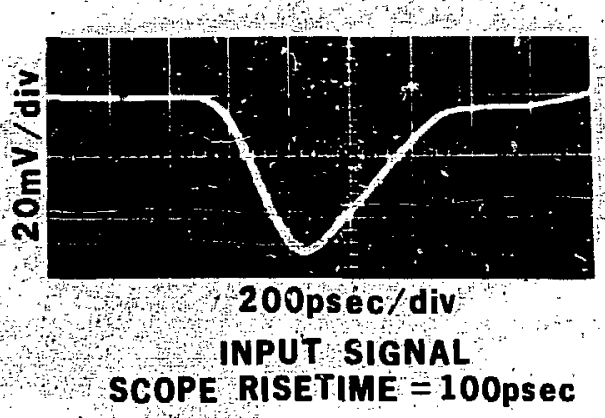

Fig. 3A

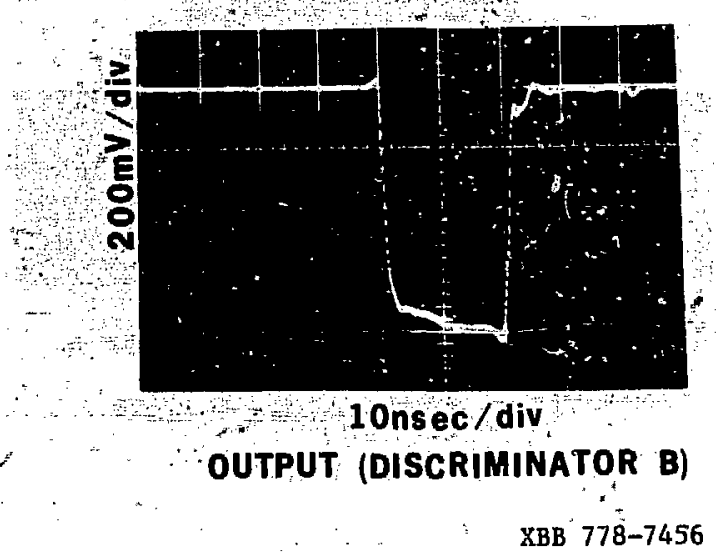

Fig. 3B 


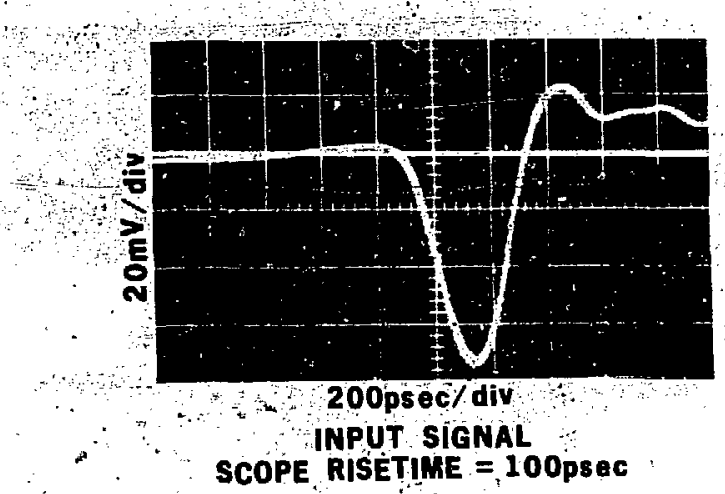

Fig. $4 A$

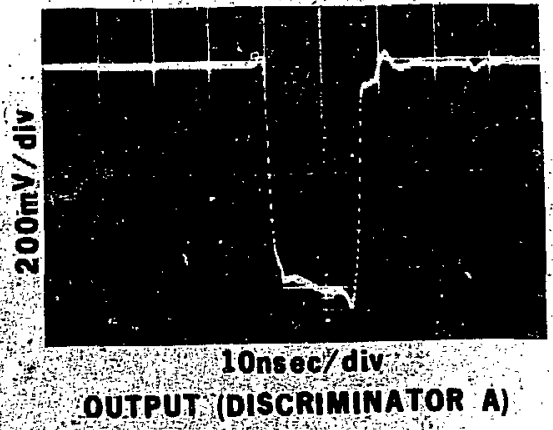

$\mathrm{XBB} 778-7457$

W 


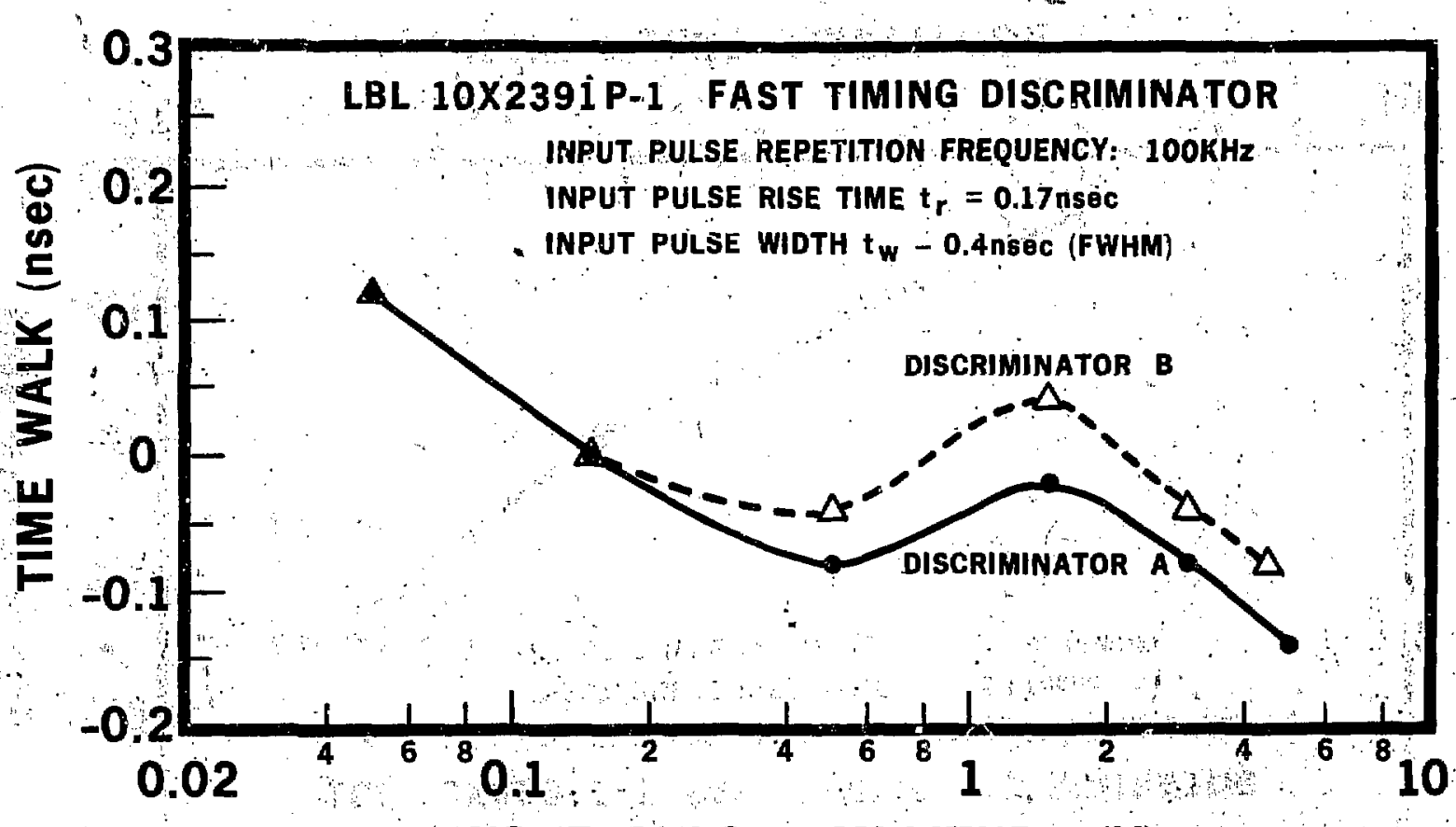
INPUT PULSE AMPLITUDE (V) 
䣷

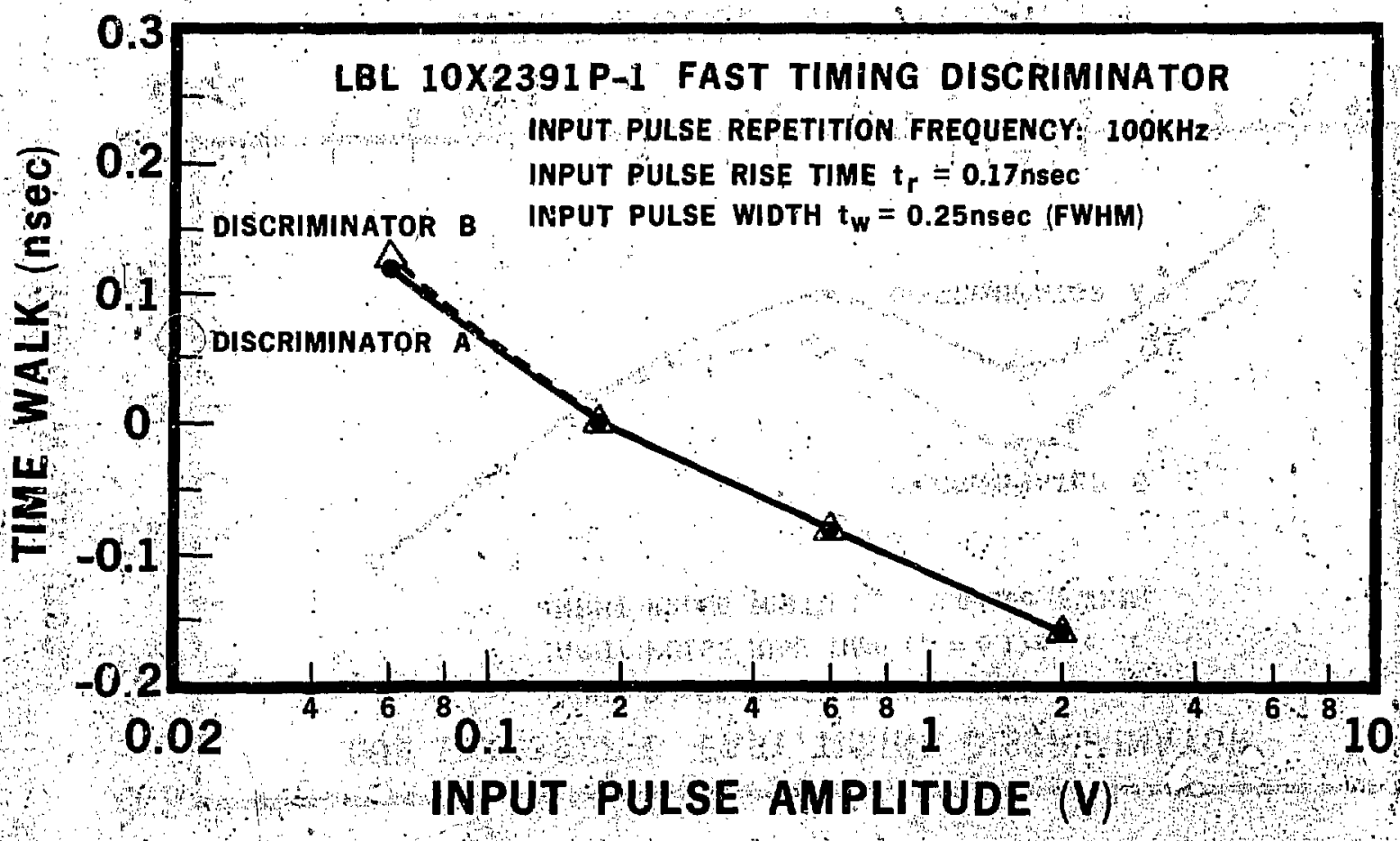




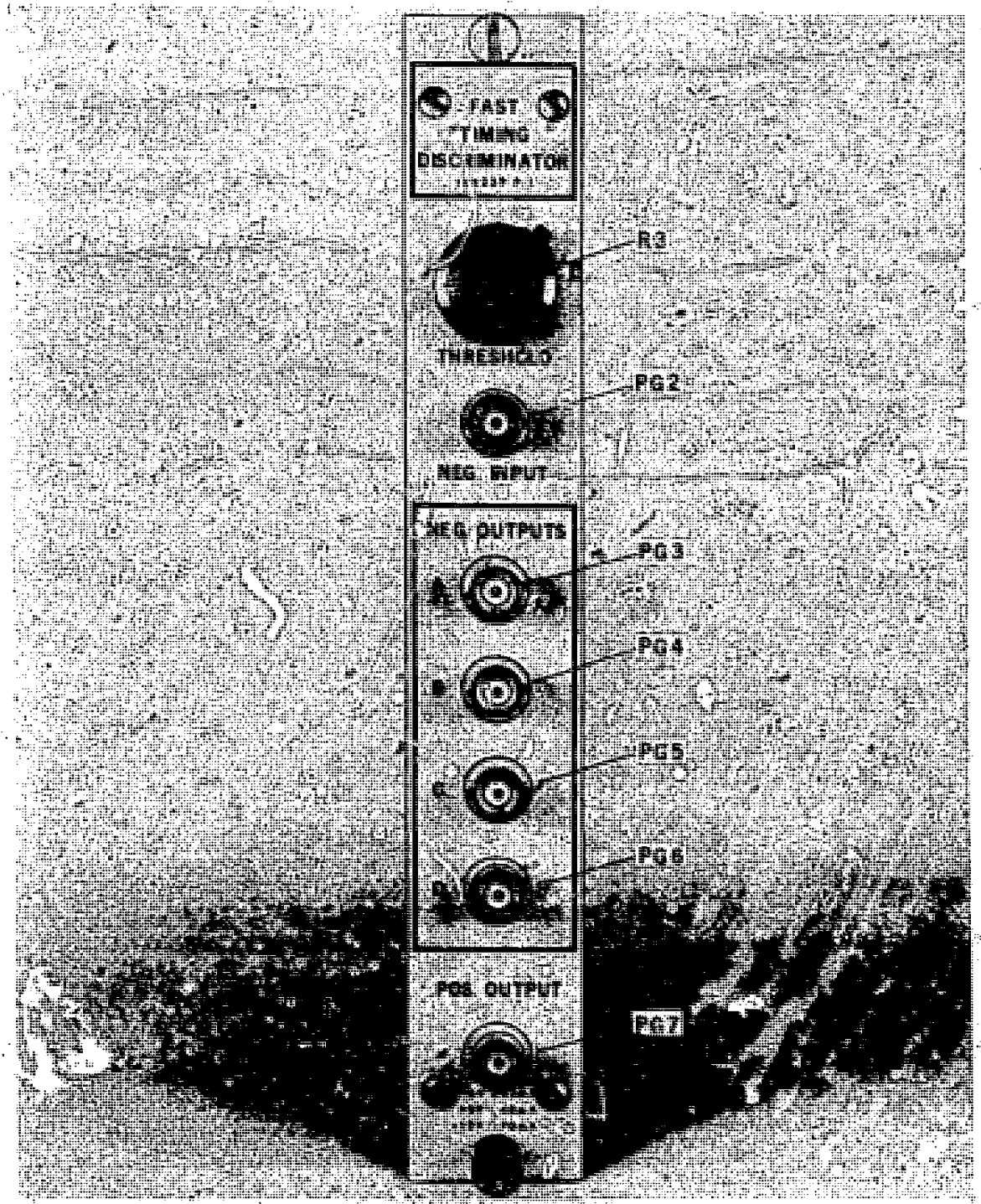




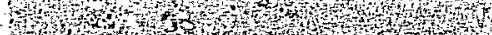
Why , Whtw

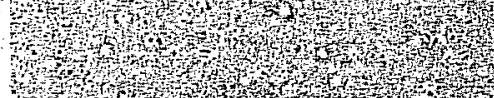

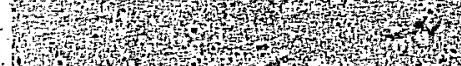

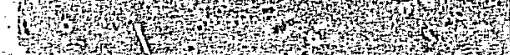

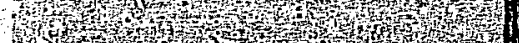
H.

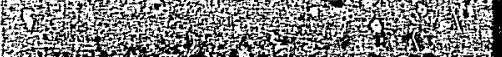

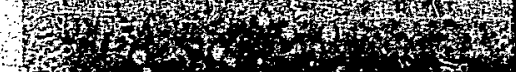
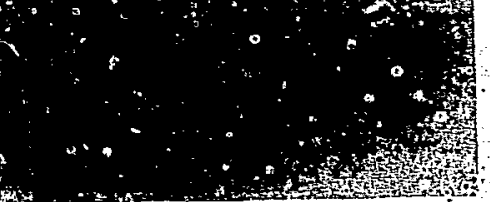

\section{.}




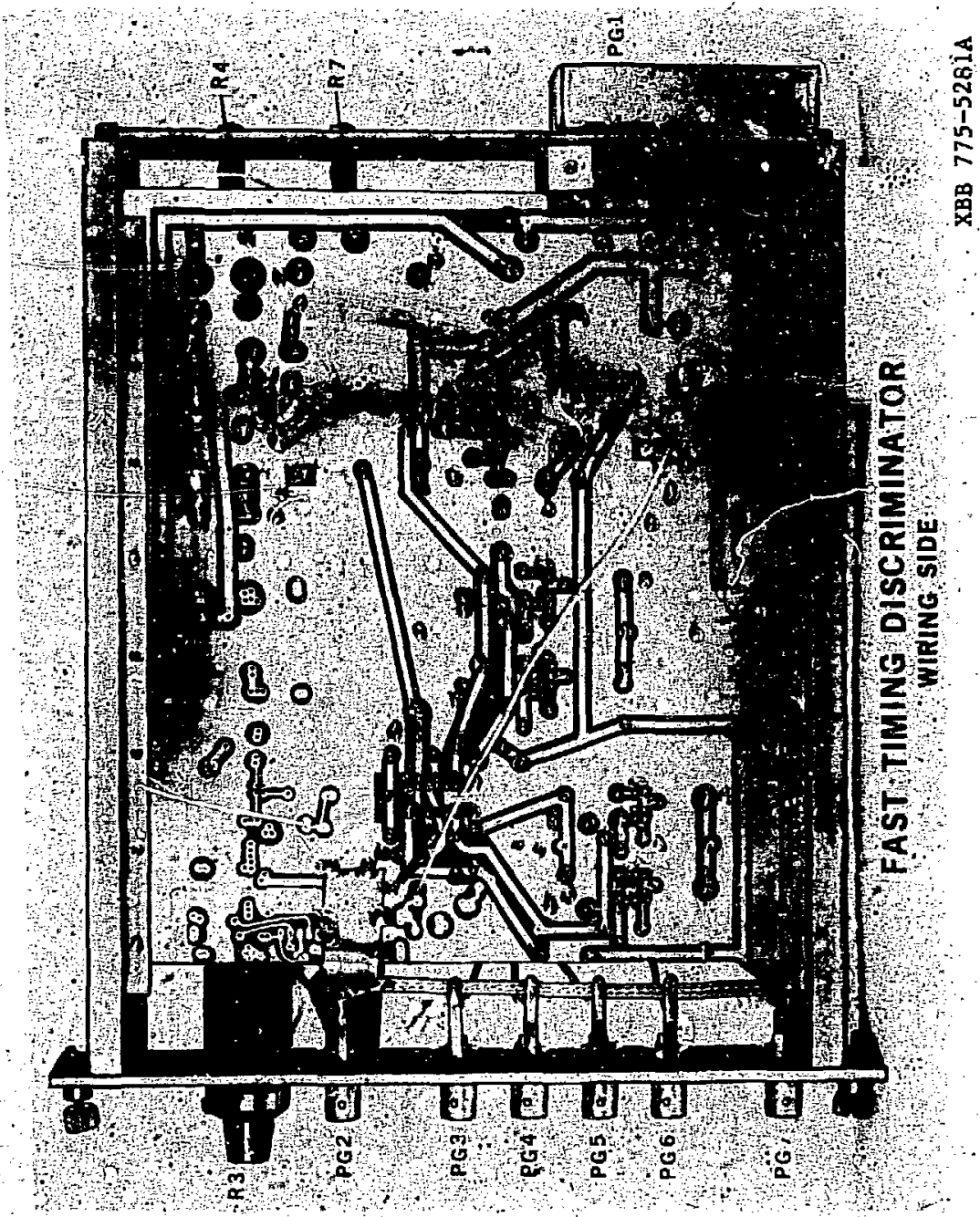

Fig 9 


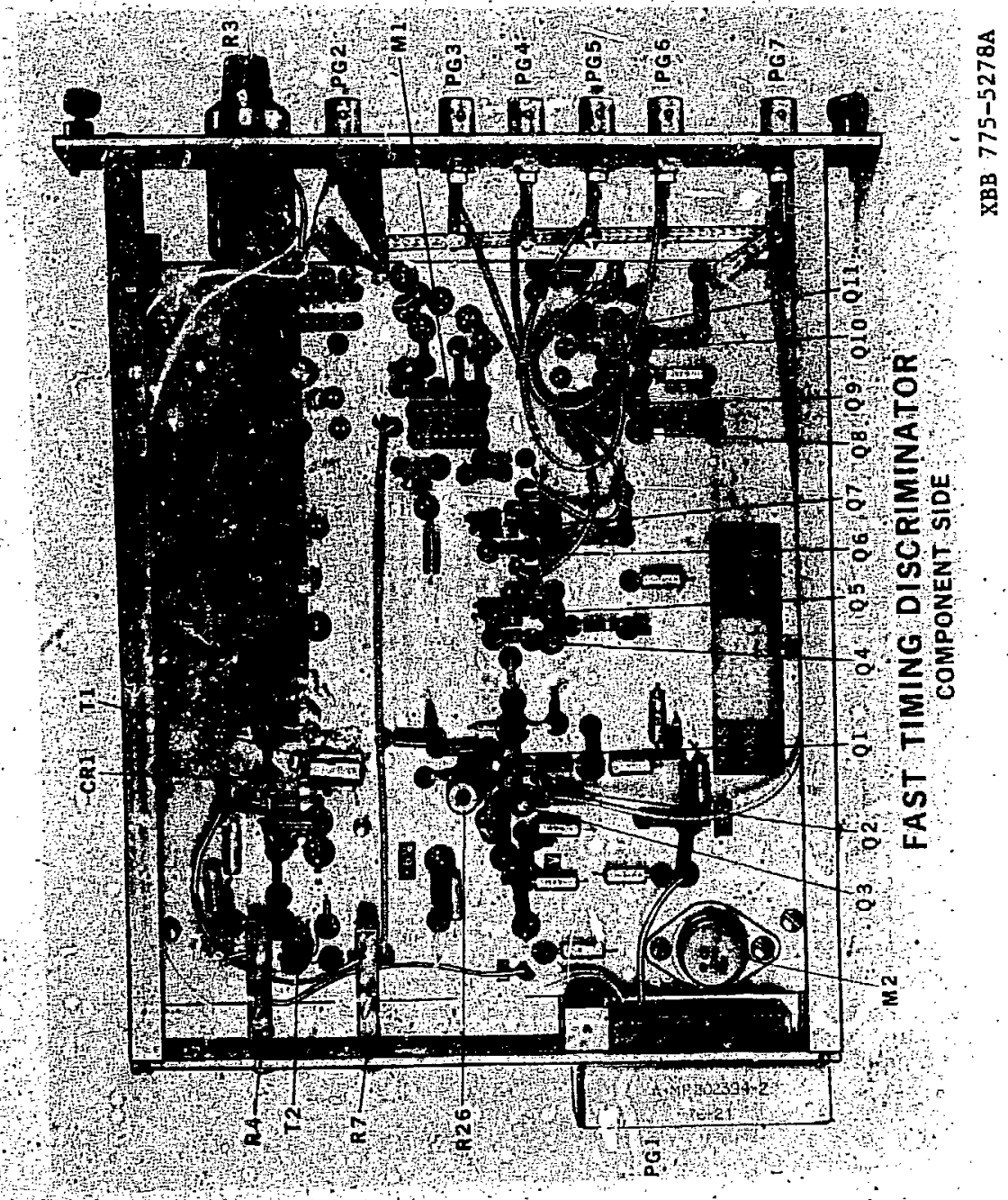

Fig. 10 


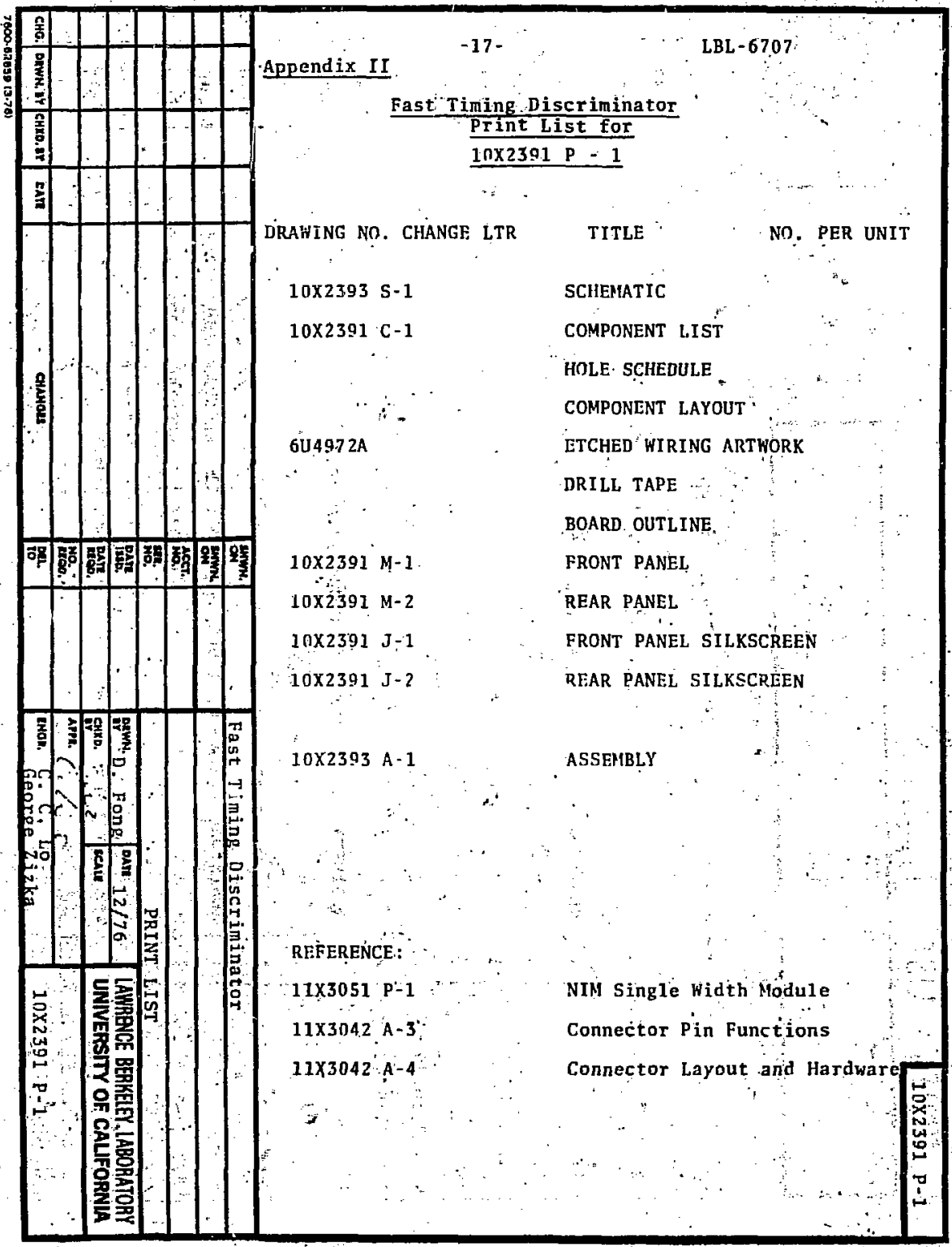




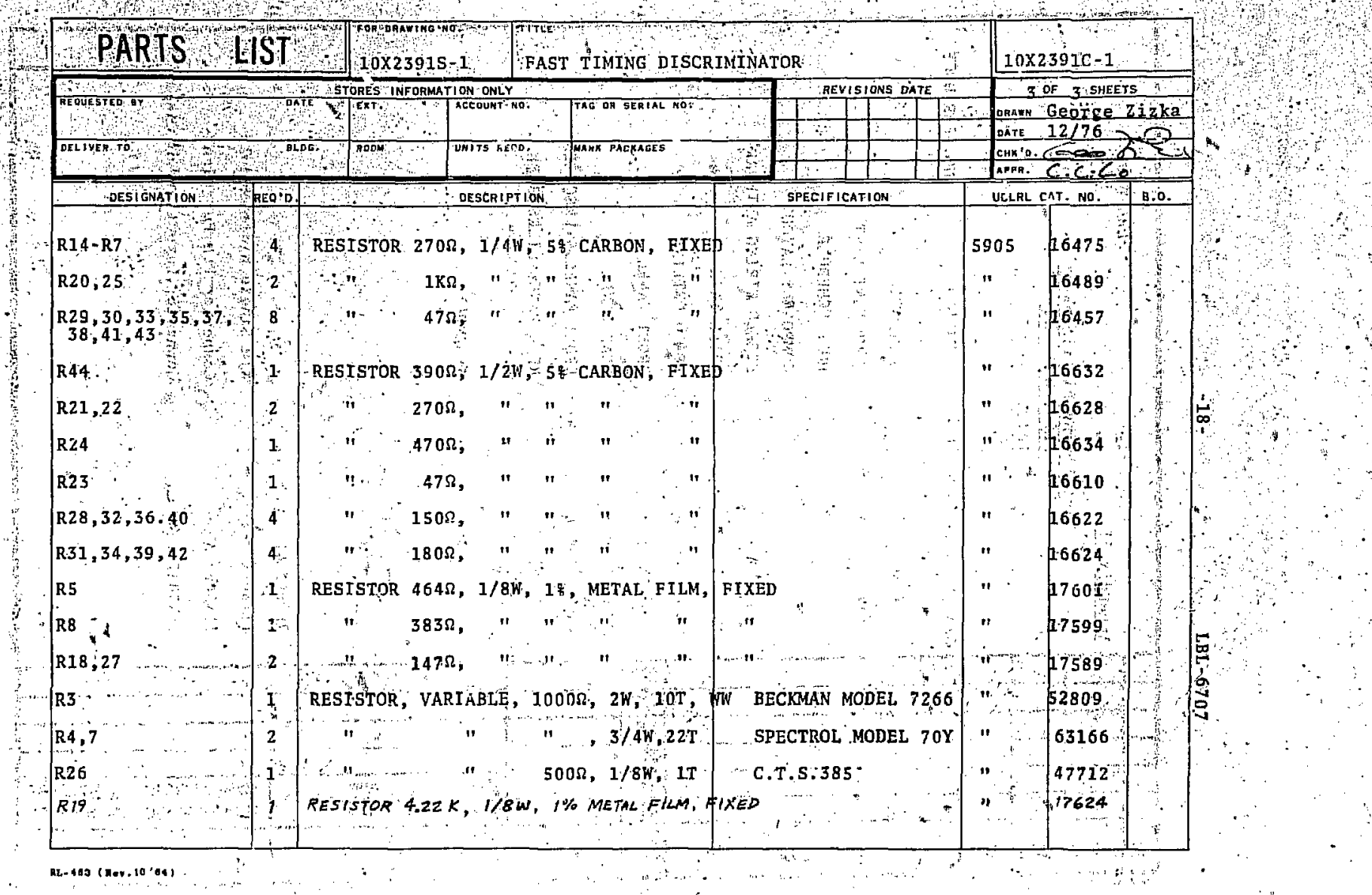




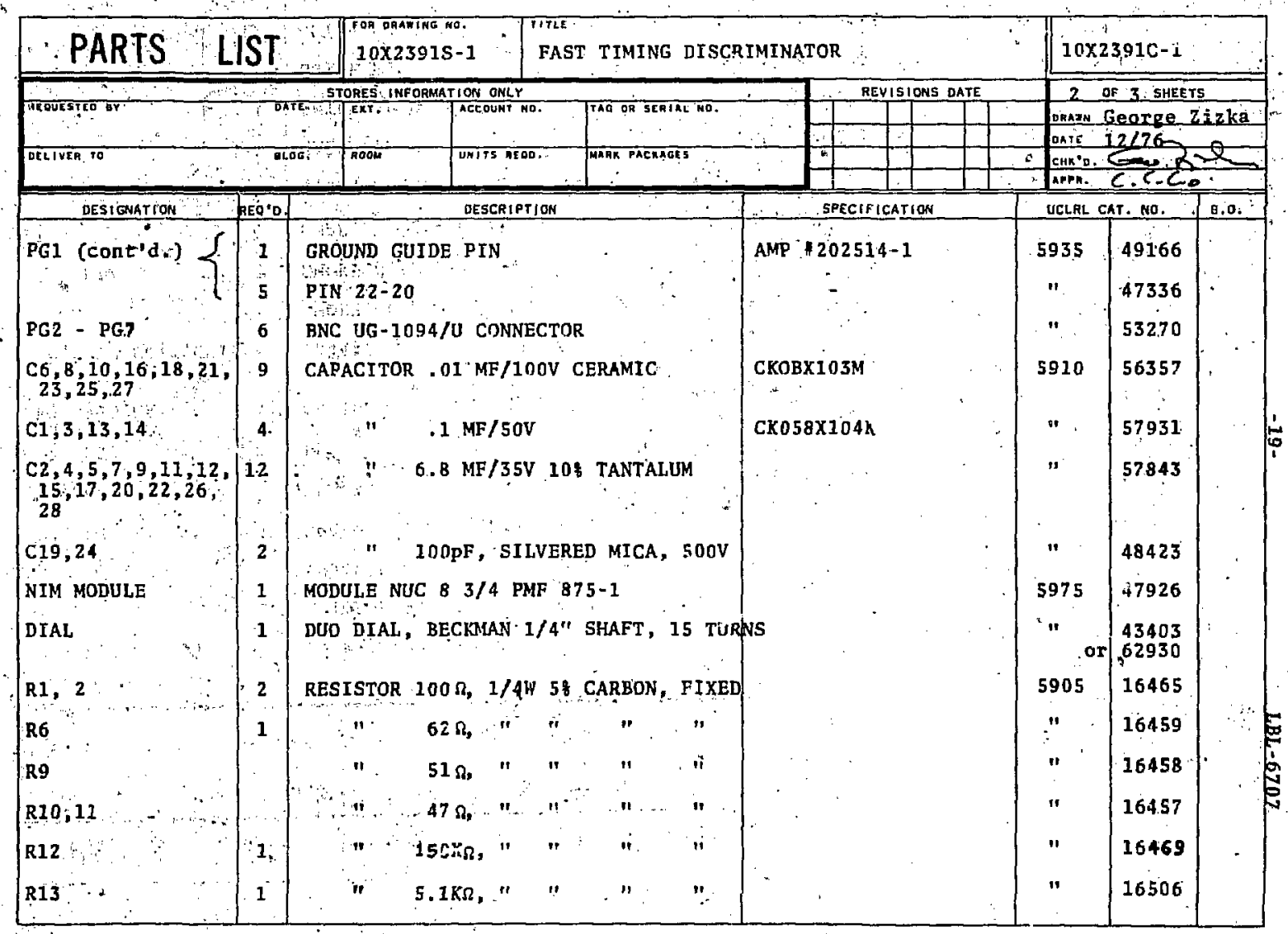

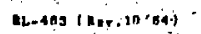




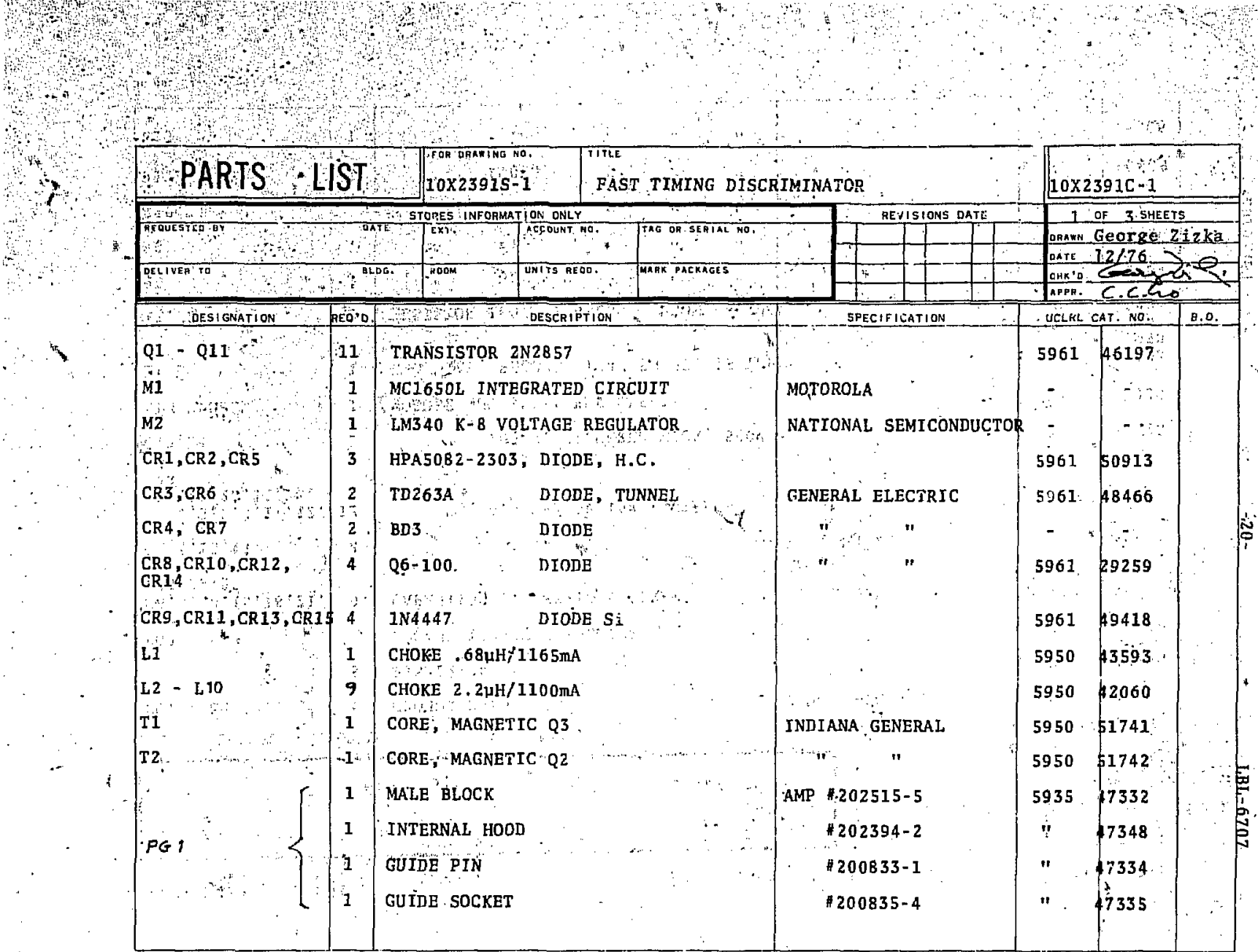

